\title{
Fruit flies modulate passive wing pitching to generate in-flight turns
}

\author{
Attila J. Bergou ${ }^{1}$ * Leif Ristroph ${ }^{1}$, John Guckenheimer ${ }^{2}$, Itai Cohen ${ }^{1}$, and Z. Jane Wang ${ }^{3}$ \\ ${ }^{1}$ Department of Physics, Cornell University, Ithaca, New York 14853, USA \\ ${ }^{2}$ Department of Mathematics, Cornell University, Ithaca, New York 14853, USA \\ ${ }^{3}$ Theoretical and Applied Mechanics, Cornell University, Ithaca, New York 14853, USA
}

(Dated: October 25, 2018)

\begin{abstract}
Flying insects execute aerial maneuvers through subtle manipulations of their wing motions. Here, we measure the free flight kinematics of fruit flies and determine how they modulate their wing pitching to induce sharp turns. By analyzing the torques these insects exert to pitch their wings, we infer that the wing hinge acts as a torsional spring that passively resists the wing's tendency to flip in response to aerodynamic and inertial forces. To turn, the insects asymmetrically change the spring rest angles to generate rowing motions of their wings. Thus, insects can generate these maneuvers using only a slight active actuation that biases their wing motion.
\end{abstract}

To generate the vertical force necessary to sustain flight, small insects must beat their wings hundreds of times per second. Under this constraint, how do they manipulate these fast wing motions to induce flight maneuvers? Although recent studies have made progress addressing how wing motions generate aerodynamic forces $[1,2,3,4]$, understanding how the wing motions themselves arise and what control variables govern them remains a challenge. To address these questions, we analyze the torques freely-flying fruit flies (D. melanogaster) exert to move their wings. Specifically, we elicit sharp free-flight turns from these flies and measure their wing and body kinematics. By using a model of the aerodynamic forces on flapping wings, we extract the torques the insects exert to generate the wing motions. From these torques, we construct a mechanical model of the wing rotation joints that demonstrates how the interplay of aerodynamic, inertial and biomechanical forces generate the wing kinematics. Finally, we connect this model to the turning dynamics of flies and describe the wing actuation mechanism that unifies these maneuvers.

To quantify turning kinematics in fruit flies, we first use three orthogonal cameras to capture their free flight maneuvers at 8000 frames per second or about 35 frames for each wing beat. We then reconstruct the threedimensional wing and body motion of the flies from these videos using the motion tracking techniques described in [5]. The body kinematics, described by the centroid coordinates and three Euler angles - yaw, $\phi_{b}$, body pitch, $\theta_{b}$, and roll, $\psi_{b}$, are shown in Fig. 1 A and visualized in Fig. 1 B. During the level flight, the fly performs a $120^{\circ}$ turn in $80 \mathrm{~ms}$, or 18 wing beats.

To induce such a turn, the insect generates differences between the motion of its left and right wings. We quantify these changes by plotting in Fig. 1 $\mathrm{1C}$ the time course of three Euler angles - stroke, $\phi$, deviation, $\theta$, and wing pitch, $\psi$ - that describe the orientation of the wings relative to the hinges they rotate about [6]. A three dimensional representation of a typical wing stroke is shown in Fig. 1D. Remarkably subtle asymmetries between the pitch angles of the wings drive the turn. For a discus- sion of why the other observed asymmetries produce a negligible effect on the yaw dynamics see 7]. To quantify the wing pitch asymmetry that induces the maneuver we phase-average [8] six consecutive strokes from $t=10$ $\mathrm{ms}$ to $30 \mathrm{~ms}$, during which these asymmetries are most prominent. We find that the symmetric front- and backstrokes of the left wing result in canceling drag forces and no net torque on the fly. In contrast, the right wing beats with mid-stroke angles of attack $\alpha=49^{\circ}$ during the front- and $\alpha=40^{\circ}$ during the back- stroke. The $9^{\circ}$ difference in $\alpha$ results in a net drag force towards the back of the fly and a torque that turns the fly clockwise. Thus, by varying wing pitch and consequently angle of attack, insects row through the air to perform a turn.

To elucidate how the fly controls its wing pitch, we invert the equations of motion of the wings to determine the torque the fly exerts at the wing hinge,

$$
\vec{\tau}_{i}=\mathbf{I}_{w} \cdot \dot{\vec{\omega}}-\vec{r} \times m_{w} \vec{a}-\vec{\tau}_{a} .
$$

From kinematic data, we determine the rotational acceleration $\dot{\vec{\omega}}$ and acceleration $\vec{a}$ of the wing centroid. We also measure the wing mass $m_{w}$ and use morphological measurements of fruit fly wings to estimate the wing moment of inertia $\mathbf{I}_{w}$ and center of mass to hinge vector $\vec{r}$ [9]. The aerodynamic torque about the wing hinge, $\vec{\tau}_{a}$, is calculated using a quasi-steady model detailed in [7, 10]. From Eq. (1), we determine the torque component flies exert to pitch their wings, $\tau_{p}$, during the turn and during steady flight.

Previous studies have suggested that the pitching torques result from torsional deformation of the wing hinge and simply resist the tendency of aerodynamic and inertial forces to flip the wing [9, 11, 12, 13, 14, 15]. To integrate these ideas into a dynamical model for the pitching torque, we plot $\tau_{p}$ versus $\psi$ in Fig. 2A for the final 9 consecutive strokes associated with steady flight in Fig. 1C. The torque data traces out an elliptical curve whose major axis has a negative slope. This negative correlation indicates that when the wing angle deviates from approximately $90^{\circ}$, the hinge produces a restoring torque. The area enclosed by the ellipse indicates the energy dis- 
A

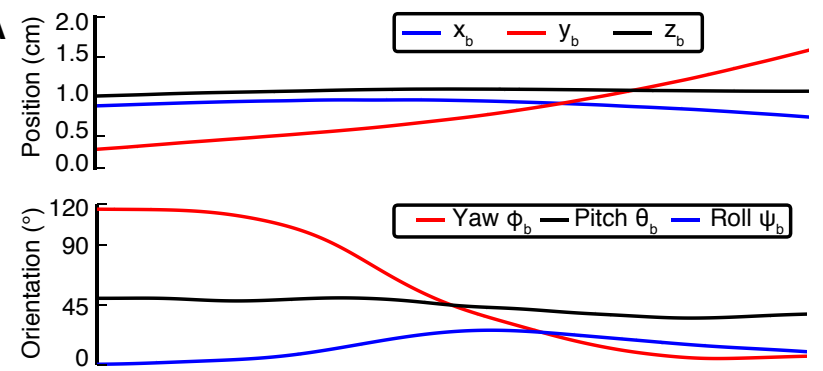

C
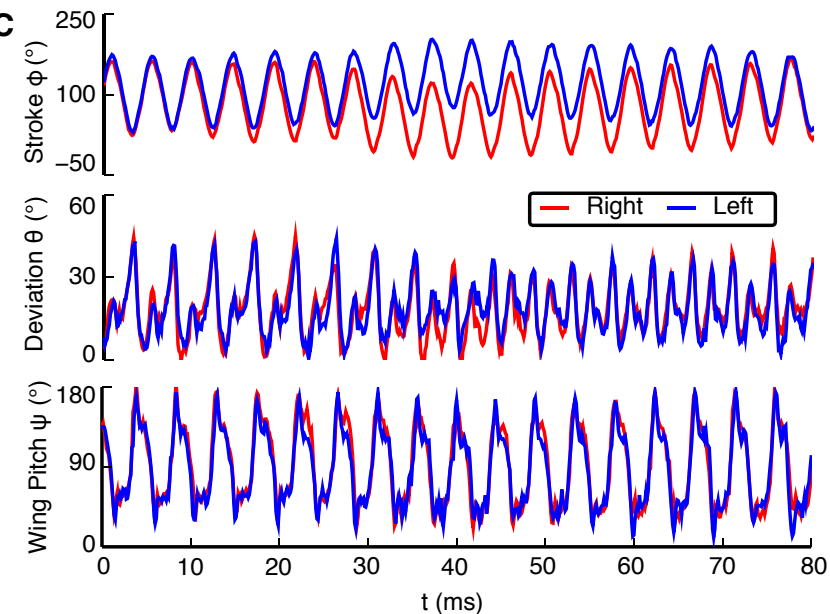

B
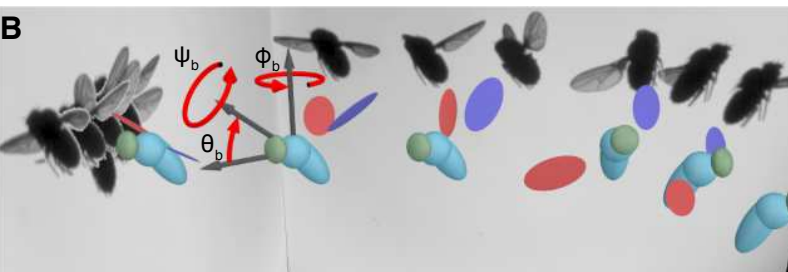

$\stackrel{\mathrm{mm}}{\longrightarrow}$

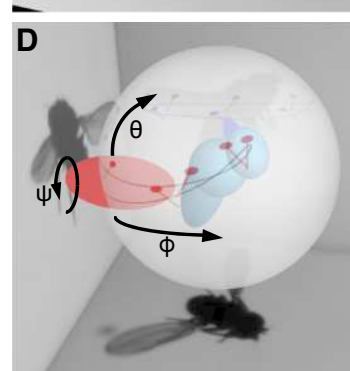

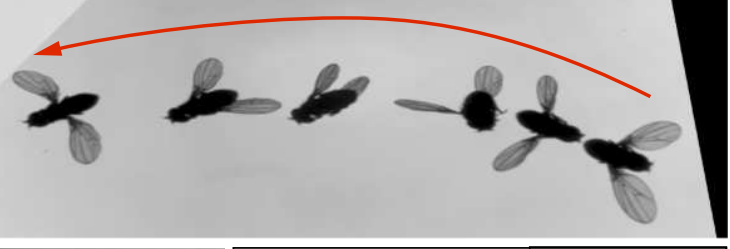

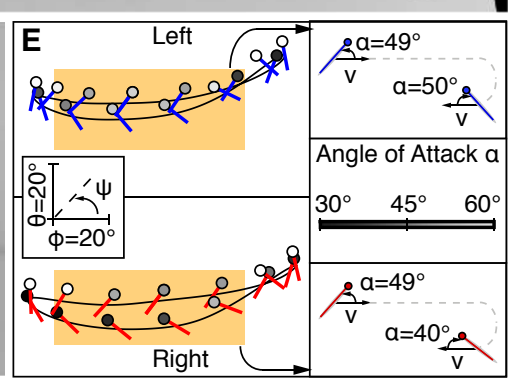

FIG. 1: (A) Measured fruit fly body position and orientation versus time. (B) Phases of motion of a fruit fly performing a $120^{\circ}$ turn. The three panels show 6 of 821 frames recorded by high speed videography, while the corresponding reconstructed kinematics are visualized using the model flies. Labeled on the fifth phase are the Euler angles that quantify the fly's orientation. (C) Measured fruit fly wing orientation relative to body versus time. (D) The trajectory and orientation of wing chords during flapping stroke. The red and blue lines on the globe depict the chord's orientation at equal time snapshots, with the ball depicting the wings leading edge. Labeled on the globe are the Euler angles used to quantify wing orientation. (E) The unwrapped ball-and-stick diagram highlights the $9^{\circ}$ asymmetry in the mid-stroke angle of attack that causes the maneuver.

sipated by the hinge as it pitches the wing. These data suggest the hinge acts like a damped torsional spring,

$$
\tau_{p}=-\kappa\left(\psi-\psi_{0}\right)-C \dot{\psi}
$$

where the parameters $\kappa, C$, and $\psi_{0}$ correspond, respectively, to the torsion constant, damping constant and the rest angle of the torsional spring. We fit this model to all 9 wing strokes, finding values $\kappa=91 \pm 9 \mathrm{pN} \mathrm{m} /{ }^{\circ}$, $C=39 \pm 12 \mathrm{fN} \mathrm{m} \mathrm{s} /{ }^{\circ}$, and $\psi_{0}=90 \pm 1^{\circ}$ that account for $95 \%$ in the variance of the pitching torque (Fig. 2A).

To determine how the wing pitch is actuated differently during the turn, we repeat the above analysis for the 6 strokes that induce the maneuver. We find that Eq. (2) also accounts for the exerted torques during these wing beats. We compare the phase-averaged torque for the 6 asymmetric strokes with the phase-averaged torque for the 9 symmetric steady strokes in Fig. 2B. We find that the two loops are shifted horizontally with respect to each other, indicating a change in $\psi_{0}$, the wing's rest angle. In fact, by plotting the values for $\kappa, C$ and $\psi_{0}$ as a function of time, we show that only $\psi_{0}$ varies significantly throughout the maneuver (Fig. 3A-C). The steadiness of $\kappa$ and $C$ suggests that these parameters correspond to material properties of the wing hinge. This interpretation is supported by the fact that the value of the spring constant, $\kappa$, agrees with scaled estimates for the torsion constants taken from measurements on the wings of house flies [12]. Comparison of the parameter values with the fly's yaw dynamics in Fig. 3D indicates that, to induce the clockwise turning maneuver (orange shading), the insect increases the $\psi_{0}$ of the right wing relative to the left by about $15^{\circ}$ for 6 strokes. We find that for the two subsequent wing beats (blue shading) the insect decreases the $\psi_{0}$ of the right wing relative to the left by about $10^{\circ}$. This reversal in the sign of $\Delta \psi_{0}=\psi_{0}^{(l)}-\psi_{0}^{(r)}$ indicates that the insect is generating a counter-clockwise torque that slows its yaw velocity. In the final 9 wing strokes (gray shading), the $\psi_{0}$ values for the left and right wings are nearly equal so that no active torque is generated.

To validate that the yaw dynamics of the fly derives from changes to the relative rest angle of the wings, $\Delta \psi_{0}$, we simulate the coupled wing-body dynamics in a model fly. The driving of each wing is simulated by prescribing its stroke and deviation angles. We then determine the fly's yaw angle and wing pitch angles from the interaction of the spring model in Eq. (2) with the 

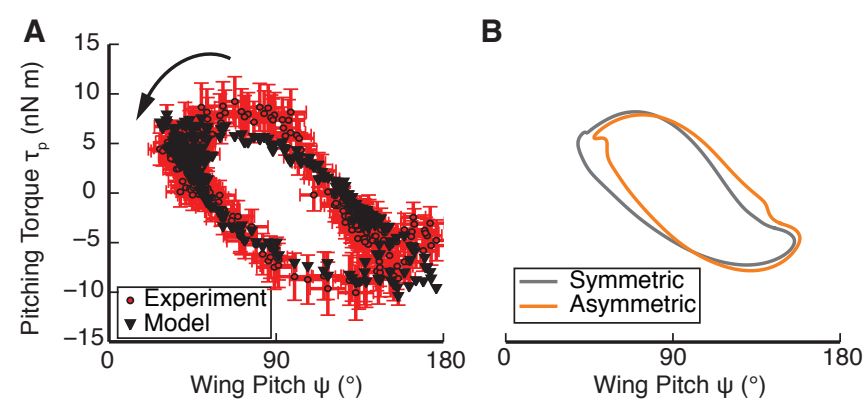

FIG. 2: (A) The pitching torque $\tau_{p}$ versus the wing pitch $\psi$ for the wing beats associated with steady flight. Red circles represent instantaneous values that correspond to frames of the flight sequence. Black triangles are the result of fitting $\tau_{p}$ with a damped torsional spring model. The counter-clockwise direction of propagation indicates that the motion is damped. (B) The phase-averaged $\tau_{p}$ versus $\psi$ for strokes associated with symmetric wing movements (gray trajectory) compared to asymmetric wing movements (orange trajectory).

aerodynamic forces on the wings [7]. To form a minimal model of the turn, the variables $\kappa$ and $C$ are held constant (dashed lines in Figs. 3A,B), $\theta$ is set to zero, and $\phi$ is driven sinusoidally with flapping amplitude and frequency that match the experiments. The rest angle, $\psi_{0}$, for the left and right wings are prescribed, respectively, by the blue and red dashed lines in Fig. $3 \mathrm{C}$, which capture the observed trends. Further, we ensure that locally the area between the dashed lines matches the area enclosed between the two experimental curves. In Fig. 3. we compare the observed yaw dynamics of the insect to those predicted by our minimal turning model. We find that the turn predicted by the model fly (dashed line) quantitatively matches experimental measurements (solid line) capturing the total accumulated yaw angle and time-course of the turn. In Fig. $3 \mathrm{E}$, we compare the measured wing pitch with our model predictions (insets) for the three regions that correspond to $\Delta \psi_{0}<0$, $\Delta \psi_{0}>0$ and $\Delta \psi_{0} \approx 0$. We find that the minimal model recovers the average measured wing pitch asymmetries, $\Delta \psi$, in these three regions, but does not reproduce the detailed sub-wing beat variations in $\psi$. When we extend our simulation to prescribe $\phi$ and $\theta$ of the wings as measured experimentally, these details are also reproduced [7]. The fact that the turning dynamics of the fly are predicted by the minimal model and are insensitive to the details of the stroke and deviation angles of the wing beat strongly indicates that the turn is controlled by $\Delta \psi_{0}$. This finding is further underscored by the fact that simulation of experimentally measured $\phi$ and $\theta$ angles without asymmetries in the rest angle, $\Delta \psi_{0}=0$, does not lead to asymmetric wing pitch angles and therefore does not yield a turn [7].

Taken together our findings show that as flies flap their wings back and forth, the wing hinges resist the tendency

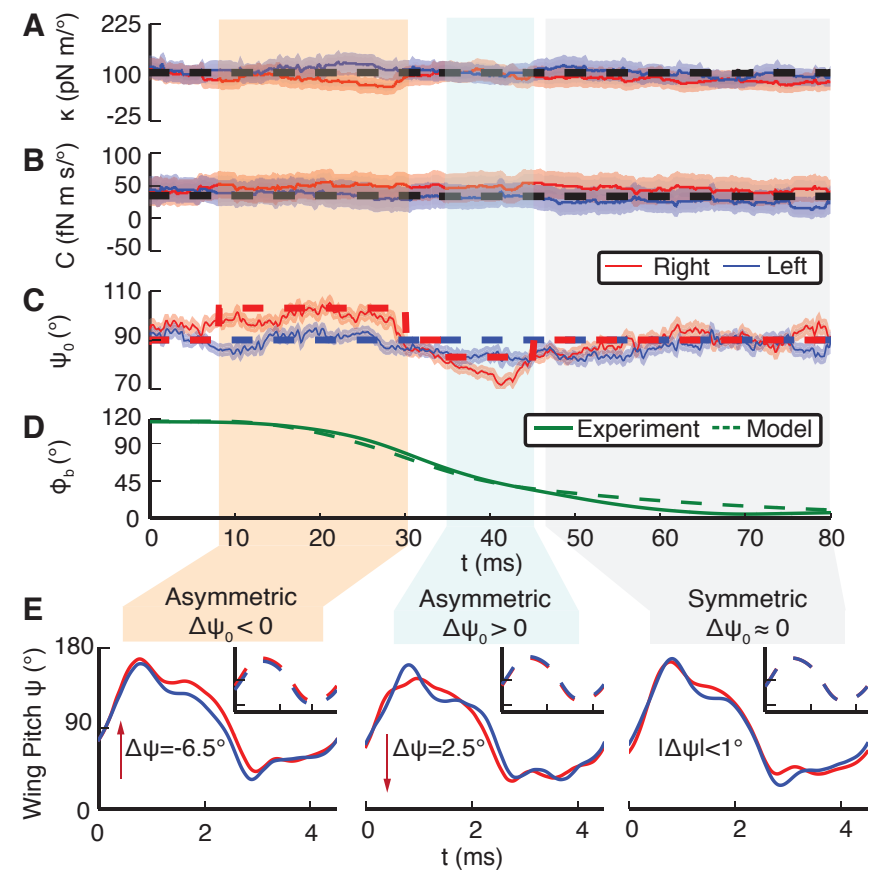

FIG. 3: (A) $-(\mathbf{C})$ Torsional spring parameters versus time extracted with error bars from the experiment data. (D) Yaw angle versus time. The solid-line corresponds to experimental measurements and dashed-line to simulation of a model fly that flaps with zero deviation angle and a sinusoidal stroke angle with amplitude and frequency that match the measured values. In the simulation, $\kappa$ and $C$ are held constant as in (A) and (B) and $\psi_{0}$ is varied as the dashed lines in (C) (E) Phase-averaged pitch of the right and left wings, $\psi$, for one complete stroke in the three labeled regions. Solid lines are experimental measurements, while dashed lines in the insets are corresponding strokes from the model fly. The shift $\Delta \psi$ in the three regions are $6.5^{\circ}$ (orange $\Delta \psi_{0}<0$ ), $2.5^{\circ}$ (blue $\Delta \psi_{0}>0$ ), and $<1.0^{\circ}$ (gray $\left|\Delta \psi_{0}\right|<1^{\circ}$ ). The corresponding $\Delta \psi$ for the model fly are $8^{\circ}, 2.9^{\circ}$ and $0^{\circ}$, respectively.

of aerodynamic and inertial forces to flip the wings. In steady flight, this passive mechanism causes the pitching of insect wings and results in front- and back- strokes with nearly symmetric angles of attack. The rest angles of the wings act as control levers that break this symmetry (Fig. 4A). This model reconciles how insects combine active and passive modulation of their wing kinematics to control flight [9, 11, 12, 13, 16, 17]. Our simulations show that the shift in relative rest angles produces a linear shift between the wing pitch angles so that $\Delta \psi \approx \mu \Delta \psi_{0}$ (Fig. (4B). We find from experimental analysis of 147 distinct wing strokes that $\mu=0.51 \pm 0.03$ which compares well with simulations of the sinusoidally flapping model fly where $\mu=0.6[7]$. The asymmetry in wing pitch angles causes asymmetric drag forces on the wings, which are related to the fly yaw dynamics by:

$$
I_{b} \ddot{\phi}_{b}+2 \bar{\omega} C_{\tau} \dot{\phi}_{b}=2 C_{\tau} \bar{\omega}^{2} \Delta \psi,
$$

where $I_{b}$ is the moment of inertia of the fly about the yaw 

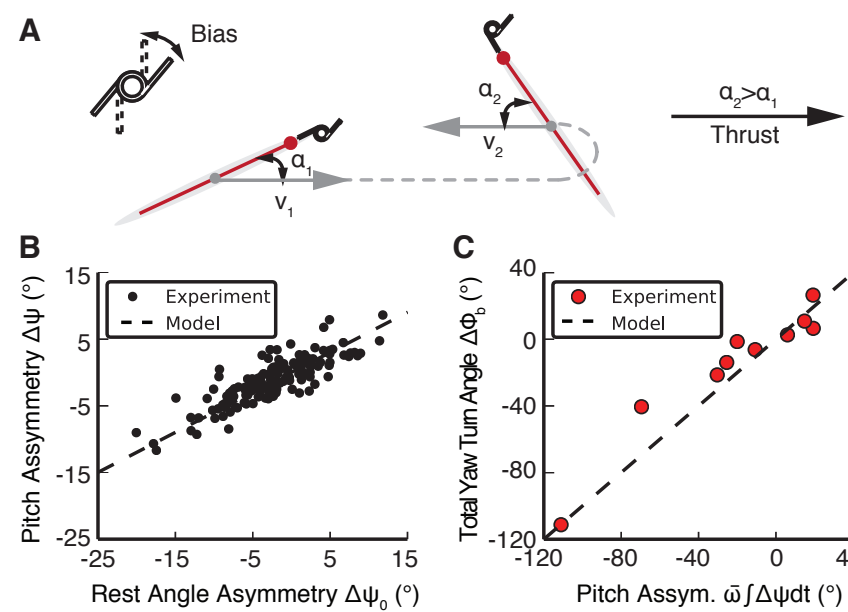

FIG. 4: (A) The minimal model of turning dynamics: the wings are driven with a sinusoidal stroke, while yaw and wing pitch are determined by aerodynamic, inertial, and spring torques. Biasing the rest angle of the spring, produces an asymmetric stroke that generates thrust. A difference in rest angles, $\Delta \psi_{0}$, of the left and right wings induces a turn. (B) The average values of $\Delta \psi$ versus $\Delta \psi_{0}$ for 147 strokes (black points). We find $\Delta \psi=\mu \Delta \psi_{0}$, where $\mu=0.51 \pm 0.03$ from experiment, and $\mu=0.6$ from simulations of the minimal turning model. Measurement errors are on the order of the spread of the data and are omitted for clarity. (C) The total turn angle of the fly versus cumulative pitch asymmetry. Measurements of ten turns (red dots) are compared with prediction of the minimal turning model (dashed line). Measurement errors are on the order of the marker size and are omitted for clarity.

axis, $C_{\tau}$ is a parameter that depends on the wing drag coefficient and geometry, $\bar{\omega}$ is the average angular velocity of the wings, and $\phi_{b}$ is the yaw of the body [7]. When $\Delta \psi=0$, Eq. (3) reduces to the passively damped yaw motion characteristic of flapping flight [18, 19]. When $\Delta \psi \neq 0$, the fly generates a driving torque that actively yaws the fly. By integrating Eq. (3) over time, we find the accumulated yaw,

$$
\Delta \phi_{b}=\bar{\omega} \int \Delta \psi d t \approx \bar{\omega} \mu \int \Delta \psi_{0} d t .
$$

We confirm that Eq. (3) accounts for the observed yaw dynamics of flies by plotting $\Delta \phi_{b}$ versus $\bar{\omega} \int \Delta \psi d t$ for 10 turning maneuvers in Fig. $4 \mathrm{C}$. We find that the yaw angles of the turns are in excellent agreement with the prediction of Eq. (4). Thus, by changing the strength and duration of the asymmetry in wing rest angles, flies can accurately control their turn angle.

In all forms of locomotion - aquatic, terrestrial, and aerial - animals take advantage of mechanical properties of their bodies to simplify the complex actuation necessary to move [20, 21, 22]. Here, we find a concrete example of how this simplification occurs as fruit flies modulate their wing motion to generate maneuvers. The mechanical properties of the wing hinge appear to be fine-tuned to enable insects to modulate their wing pitch through only slight active actuation. The spring-like behavior of the wing hinge also connects the timescale of a turning maneuver with the timescale of the wing actuation. Our model predicts, that flight muscles of flies can act over several wing beats to bias the pitch of the wings and yet generate the sub wing-beat changes in wing motion that aerodynamically induce the maneuver. Finally, because animals over a wide range of length scales experience similar rotational dynamics [19], the simple mechanism used by fruit flies may be quite general and should likewise simplify the control of flapping flying machines.

We thank Andy Ruina, Gordon Berman, Kirk Jensen, Song Chang for helpful discussions. This work is supported by NSF.

* Electronic address: ajb78@cornell.edu

$\dagger$ Electronic address: zw24@cornell.edu

[1] S. Fry, R. Sayaman, and M. H. Dickinson, Science 300, 495 (2003).

[2] C. P. Ellington, Phil. Trans. R. Soc. B 305, 1 (1984).

[3] S. P. Sane, J. Exp. Biol. 206, 4191 (2003).

[4] Z. J. Wang, Annu. Rev. Fluid. Mech. 37, 183 (2005).

[5] L. Ristroph, G. J. Berman, A. J. Bergou, Z. J. Wang, and I. Cohen, J. Exp. Biol. 212 (2009).

[6] R. J. Wootton, Annu. Rev. Entomol. 37, 113 (1992).

[7] See EPAPS Document No. ? for detail of the experiment, simulations, and explicit calculations related to the paper. For more information on EPAPS, see http://www .aip.org/pubservs/epaps.html

[8] S. Revzen and J. M. Guckenheimer, Phys. Rev. E 78, 051907 (2008).

[9] A. R. Ennos, J. Exp. Biol. 140, 161 (1988).

[10] U. Pesavento and Z. J. Wang, Phys. Rev. Lett. 93, 144501 (2004).

[11] A. J. Bergou, S. Xu, and Z. J. Wang, J. Fluid Mech. 591, 321 (2007).

[12] A. R. Ennos, J. Exp. Biol. 140, 137 (1988).

[13] J. A. Miyan and A. W. Ewing, J. Exp. Biol. 136, 229 (1988).

[14] D. Ishihara, T. Horie, and M. Denda, J. Exp. Biol. 212, 1 (2009).

[15] A. M. Mountcastle and T. L. Daniel, Exp. Fluids (2009).

[16] J. B. Pettigrew, Animal Locomotion or Walking, Swimming and Flying (Henry S. King, London, 1873).

[17] E.-J. Marey, Movement (William Heinemann, London, 1895).

[18] T. Hesselberg and F.-O. Lehmann, J. Exp. Biol. 210, 4319 (2007).

[19] T. L. Hedrick, B. Cheng, and X. Deng, Science 324, 252 (2009).

[20] M. H. Dickinson, C. T. Farley, R. J. Full, M. A. R. Koehl, R. Kram, and S. Lehman, Science 288, 100 (2000).

[21] J. C. Liao, D. N. Beal, G. V. Lauder, and M. S. Triantafyllou, Science 302, 1566 (2003).

[22] S. Collins, A. Ruina, R. Tedrake, and M. Wisse, Science 307, 1082 (2005). 